\title{
The Utility of a Portable Recording Device for Screening of Obstructive Sleep Apnea in Obese Adolescents
}

\author{
Daniel J. Lesser, M.D. ${ }^{1,3}$; Gabriel G. Haddad, M.D. ${ }^{1,2,3}$; Ruth A. Bush, Ph.D.3; Mark S. Pian, M.D. ${ }^{1,3}$ \\ ${ }^{1}$ Departments of Pediatrics (Division of Pediatric Respiratory Medicine) and ${ }^{2}$ Neurosciences, University of California-San Diego \\ School of Medicine, San Diego, CA; ${ }^{3}$ Rady Children's Hospital-San Diego, San Diego, CA
}

Study Objectives: The ApneaLink Plus is a portable recording device that measures air flow, respiratory effort, heart rate, and pulse oximetry. In the current study, we asked whether this device could be used to screen for obstructive sleep apnea in the pediatric population.

Methods: Sleep-laboratory polysomnography (PSG) was performed simultaneously with measurements using the portable device on obese pediatric patients referred for snoring. The obstructive apnea hypopnea index (OAHI) was calculated automatically by the device (autoscore) and manually by the investigators. Sensitivity, specificity, correlation, and receiver operating curves (ROC) were used to compare the portable device to PSG.

Results: Twenty-five subjects $(60 \%$ male, mean age $13.6 \pm$ 3.0 years, OAHI on PSG $11.8 \pm 27.1$ ) were studied. We identified a significant correlation between the OAHI of the ApneaLink autoscore and PSG (Spearman Rho $=0.886[p<0.001]$ ). Using the PSG results as standard, ROC curves comparing the ApneaLink OAHI with the PSG OAHI demonstrated high congruence. The autoscore agreement was very good at PSG
$\mathrm{OAHI}>1.5$ (area under the receiver operating curve $[\mathrm{AUC}]$ $0.965, \mathrm{OAHI}>5$ [AUC 0.937], and OAHI > 10 [AUC 1.00]). The agreement of the manual score and autoscore were essentially equivalent. The device's autoscore demonstrated high sensitivity at all cutoffs examined $(100 \%$ at $\mathrm{OAHI}>1.5,85.7 \%$ at $\mathrm{OAHI}>5$, and $100 \%$ at $\mathrm{OAHI}>10)$. The specificity increased with increasing cutoffs ( $46.2 \%$ at $\mathrm{OAHI}>1.5,83.3 \%$ at $\mathrm{OAHI}>$ 5 , and $90.0 \%$ at $\mathrm{OAHI}>10)$.

Conclusion: The ApneaLink Plus is a sensitive screening tool for evaluation of suspected OSAS in obese pediatric patients aged 9-18 years. The specificity improves with increasing OAHI cutoffs. The device detects OSAS when tested in a sleep laboratory on obese adolescents referred for symptoms of sleep related breathing disorder.

Keywords: Obstructive sleep apnea, polysomnography, ApneaLink, screening, adolescent, apnea

Citation: Lesser DJ; Haddad GG; Bush RA; Pian MS. The utility of a portable recording device for screening of obstructive sleep apnea in obese adolescents. J Clin Sleep Med 2012;8(3):271-277.

A $\mathrm{n}$ increasingly high incidence of pediatric obesity is well documented. ${ }^{1}$ The link between obesity and obstructive sleep apnea syndrome (OSAS) has, in turn, increased demand for diagnostic services aimed at identifying pediatric patients with OSAS. OSAS has been linked to poor cognitive performance, cardiovascular morbidity, and insulin resistance in adolescent populations. ${ }^{2-4}$ In adults, OSAS is associated with cardiovascular disease, cerebrovascular disease, insulin resistance, and mortality. Early identification and therapy for OSAS during childhood and adolescence likely minimizes its neurocognitive and cardiovascular complications and may enable efforts aimed at decreasing associated morbidities during adulthood. However, the availability of pediatric sleep laboratories is limited. Practitioners and families often face significant delays in diagnosis and therapy of OSAS. A preliminary assessment using a screening device in the home to identify suitable candidates for standard polysomnography would likely be a cost-effective method of decreasing wait times and improving access for high-risk patients.

The ApneaLink Plus (ResMed Corporation, Poway, CA) is a multichannel screening device that records nasal airflow by nasal pressure transducer, respiratory effort, pulse rate, and hemoglobin saturation by pulse oximetry. The utility of single-

\begin{abstract}
BRIEF SUMMARY
Current Knowledge/Study Rationale: Clinicians and families often face significant delays in diagnosis and therapy of pediatric OSAS due to limited availability of pediatric sleep laboratories. We asked whether a multi-channel screening device could be used to diagnose OSAS in obese children and adolescents referred for symptoms of sleep related breathing disorders.

Study Impact: The portable device tested in this study accurately identifies OSAS when tested in a sleep laboratory on obese adolescents. Future investigation of the accuracy of the device when used in the home will help to define further the appropriate use of multi-channel screening devices in pediatric patients.
\end{abstract}

channel screening devices used for OSAS has been suggested in a number of studies in adult subjects..$^{5-12}$ However, important differences exist between adults and children in the pathophysiology and diagnostic criteria defining OSAS. ${ }^{13,14}$ Thus, studies of portable devices in adults cannot be assumed to apply to children and adolescents.

Few studies have examined home sleep studies in children. Nixon et al. reported that home oximetry may be useful in identifying severe cases of OSAS. ${ }^{15}$ Yet, home oximetry alone likely has limited usefulness for identifying all cases of OSAS. ${ }^{16}$ Moss 
et al. published reference values for home polysomnography using a multichannel airflow device on healthy subjects without snoring. ${ }^{17}$ Less is known about the accuracy of multichannel screening devices in identifying OSAS in children. ${ }^{18}$

We asked whether a multichannel screening device could be used to screen for OSAS in a referral population of obese children and adolescents referred for symptoms of sleep related breathing disorder. We hypothesized that the device would have improved accuracy when higher OAHI cutoff levels are used to diagnose OSAS. This study compared data obtained using the ApneaLink device with data obtained during standard sleep laboratory polysomnography.

\section{METHODS}

\section{Subjects}

Twenty-nine obese (BMI $\geq 95$ th percentile for age/gender), otherwise generally healthy subjects $9-18$ years old with snoring were sequentially recruited from patients referred to the Rady Children's Hospital San Diego Sleep Laboratory. Exclusion criteria included a history of cardiac disease, craniofacial disorder, genetic syndrome, cerebral palsy, inability to initiate sleep during PSG, or use of positive airway pressure therapy. Subjects were withdrawn if they had $<2 \mathrm{~h}$ of ApneaLink data recorded. ${ }^{5}$ The University of California, San Diego institutional review board approved the study, and written informed assent/ consent was obtained from the subjects and their parents.

\section{Polysomnography}

Overnight attended PSG in our sleep center was performed on all subjects. The ApneaLink and PSG studies were performed simultaneously. PSG data were collected using the Sandman Elite Sleep Diagnostic Software System (Bakersfield, CA). Surface electrodes were connected to subjects to monitor electrocardiogram, chin electromyogram, electroencephalogram (frontal, central, and occipital), and electrooculogram. Thoracoabdominal movement was measured by piezoelectric bands, and oxygen saturation was recorded using pulse oximetry. Expired carbon dioxide was measured continuously with a sampling catheter, and leg movements were recorded. A nasal-oral thermistor was used to evaluate combined airflow (Salter Labs ThermiSense, Arvin, CA). A nasal cannula for nasal pressure measurement was connected directly to the ApneaLink recording device.

All PSGs were scored manually by a single sleep technologist and reviewed by a single investigator. Both the sleep technologist and investigator were blinded to the results of the ApneaLink device when scoring PSGs. Raw data were scored according to American Academy of Sleep Medicine criteria. ${ }^{19}$ The obstructive apnea hypopnea index (OAHI) was calculated as the number of obstructive apneas, mixed apneas, and hypopneas per hour of sleep. The total arousal index (TAI; spontaneous, movement, or respiratory) was calculated as the total number of arousals per hour of sleep.

\section{Multichannel Device}

The ApneaLink Plus is a multichannel tool designed to screen for OSAS. The device records airflow via a nasal cannula connected to a pressure transducer. A belt placed around the chest records thoracic respiratory movement and electrocardiogram. Pulse oximetry is also recorded via digital probe. Data generated by the airflow signal, respiratory effort signal, and pulse oximeter are used by the ApneaLink software to score apnea. The software defines an obstructive apnea as $80 \%$ to $100 \%$ reduction in airflow associated with respiratory effort $\geq 10 \mathrm{sec}$, and an obstructive hypopnea as $50 \%$ to $80 \%$ reduction in airflow associated with respiratory effort $\geq 10 \mathrm{sec}$. The software calculates AHI from the number of apneas (central, mixed, and obstructive) and hypopneas per hour of device recording time. For the purpose of this study, the AHI calculated by the software was recalculated to reflect the OAHI (the number of obstructive apneas, mixed apneas, and hypopneas/h of device recording time). Central apneas were recorded separately. In addition, the software reports mean oxygen saturation $\left(\mathrm{SpO}_{2}\right)$, $\mathrm{SpO}_{2}$ nadir, and oxygen desaturation index (ODI; number of desaturations $>3 \% / \mathrm{h}$ recording).

The ApneaLink software also enables the investigator to score manually the channels' output epoch by epoch. For this study, each ApneaLink study was scored manually by the same investigator based on pediatric criteria. An obstructive apnea was defined as $80 \%$ to $100 \%$ reduction in airflow for 2 respiratory cycles associated with respiratory effort; obstructive hypopnea was scored if airflow was decreased by $50 \%$ to $80 \%$ for 2 respiratory cycles in the presence of respiratory effort. Decreases in airflow that appeared to be associated with movement artifact were not scored. When scoring the ApneaLink data manually, the investigator was blinded to the results of the ApneaLink autoscore. However, because the same investigator reviewed the PSG and ApneaLink, the investigator was not blinded to the results of the PSG when the ApneaLink manual scoring was performed.

The ApneaLink software identifies epochs of the study associated with poor airflow signal as "signal too small." This occurs if $<8.5 \%$ of the average signal amplitude during inspiration is detected for a time period $\geq 2 \mathrm{~min}$. The flow evaluation time (total device recording time with adequate flow signal) and the percent of flow data removed by the device due to inadequate airflow were recorded for each subject. The automatic scoring feature excludes "signal too small" portions of the study in its analysis.

\section{Analysis}

Power analysis revealed that a correlation coefficient of 0.60 would require a sample size of 25 for a 2 -tailed $\alpha$ error of 0.05 and a $\beta$ error of 0.10 . Descriptive results were summarized as mean plus or minus the standard deviation, with the range disclosed. Correlation analysis was performed to compare the number of apneas identified by each modality. Because the data for the device and PSG were not normally distributed, Spearman Rho was used to account for non-normal distribution. Analysis was performed using OAHI cutoff values of 1.5, 5 , and 10 used to diagnose OSAS. Only subjects who had > $2 \mathrm{~h}$ of sleep recorded on the ApneaLink device were included in the data analysis. The PSG data were compared both to automatically and manually scored ApneaLink data. To validate results, receiver operating curves (ROC) were used to compare graphically sensitivity and specificity. The ROC plots sensitivity against 1 - specificity across variable OAHI values. A 
Table 1-Demographic and sleep characteristics

\begin{tabular}{|c|c|c|}
\hline Variable $n=25(15$ male, $60 \%)$ & (mean \pm SD) & Range \\
\hline \multicolumn{3}{|l|}{ Demographic } \\
\hline Age (years) & $13.6 \pm 3.0$ & $9.0-18.2$ \\
\hline $\mathrm{BMI}\left(\mathrm{kg} / \mathrm{m}^{2}\right)$ & $34.5 \pm 6.2$ & $25.1-47.0$ \\
\hline BMI (z-score) & $2.4 \pm 0.3$ & $1.7-3.0$ \\
\hline \multicolumn{3}{|l|}{ Polysomnography } \\
\hline Total Sleep Time (min) & $317 \pm 61$ & $146-408$ \\
\hline Sleep efficiency (\%) & $74.6 \pm 14.4$ & $34-92$ \\
\hline REM (\%TST) & $15.5 \pm 4.8$ & $9.1-23.8$ \\
\hline $\mathrm{OAHI}(\mathrm{n} / \mathrm{h})$ & $11.8 \pm 27.1$ & $0-126$ \\
\hline Total Arousal Index (n/h) & $13.0 \pm 12.7$ & $3.1-68.8$ \\
\hline Number Central Apneas & $3.6 \pm 8.1$ & $0-37$ \\
\hline $\mathrm{SpO}_{2}$, nadir $(\%)$ & $87 \pm 10$ & $50-95$ \\
\hline Mean $\mathrm{SpO}_{2}(\%)$ & $97 \pm 2$ & $88-100$ \\
\hline \multicolumn{3}{|l|}{ ApneaLink Portable Device } \\
\hline Recording Time (min) & $423.2 \pm 29.5$ & $371-502$ \\
\hline Flow Evaluation Time (min) & $287.1 \pm 103.7$ & $148-2004$ \\
\hline \% Flow Data Removed & $35.0 \pm 25.5$ & $3-85$ \\
\hline Autoscore OAHI (n/h) & $12.5 \pm 20.6$ & $0-83.1$ \\
\hline Manual score OAHI (n/h) & $10.3 \pm 21.1$ & $0-100.5$ \\
\hline Autoscore \# CA & $3.1 \pm 7.4$ & $0-29$ \\
\hline Manual score \# CA & $10.4 \pm 22.7$ & $0-87$ \\
\hline ODI & $9.6 \pm 13.3$ & $0-41$ \\
\hline $\mathrm{SpO}_{2}$, nadir (\%) & $85 \pm 10$ & $55-94$ \\
\hline Mean $\mathrm{SpO}_{2}(\%)$ & $96 \pm 2.3$ & $88-98$ \\
\hline
\end{tabular}

BMI, body mass index; OAHI, obstructive apnea hypopnea index; Flow Evaluation Time, time using device with adequate flow signal; \% Flow Data removed, \% of data removed by device due to inadequate airflow; CA, central apnea; ODI, oxygen desaturation index (\# desaturations $>3 \%$ / h recording).

better test displays a steeper rise in curve. This is quantified by measuring the area under the curve (AUC). An AUC of 1 represents a perfect instrument, and 0.5 denotes the absence of a relationship between the 2 devices. Pairwise comparison of ROC curves was applied to study the difference between the manual and autoscore. Based on the distribution of the data, the option of computing the estimate of the area under the ROC curve with the assumption that the distribution is non-normal was used. The sensitivity, specificity, positive predictive value, and negative predictive value were calculated at each OAHI cutoff. A p value $<0.05$ was considered statistically significant.

\section{RESULTS}

Twenty-five obese pediatric subjects (15 males) with mean BMI $z$-score $2.4 \pm 0.3$ and mean age of $13.6 \pm 3.0$ years completed the study (Table 1). The ApneaLink device records areas of the study with inadequate airflow and does not include these parts of the study in the scoring of apnea. Twenty-five of 29 enrolled subjects $(86 \%)$ had ApneaLink data with $>2 \mathrm{~h}$ of usable airflow and were included in the analysis. Among the 25 subjects, the ApneaLink autoscore recorded a mean flow evaluation period of $287 \pm 104 \mathrm{~min}$ and a mean total recording time of $423 \pm 30 \mathrm{~min}$. Thus, on average $35 \% \pm 25 \%$ of total ApneaLink recording time was not used for sleep scoring because of poor airflow signal. We observed a substantial range in the values of
Table 2-Individual comparison of OAHI across scoring modalities

\begin{tabular}{cccccc}
$\begin{array}{c}\text { Subject } \\
\text { Number }\end{array}$ & $\begin{array}{c}\text { Age } \\
\text { (years) }\end{array}$ & $\begin{array}{c}\text { Sex } \\
1\end{array}$ & $\begin{array}{c}\text { Device OAHI } \\
\text { (Manual Score) }\end{array}$ & $\begin{array}{c}\text { Device OAHI } \\
\text { (Auto Score) }\end{array}$ & $\begin{array}{c}\text { PSG } \\
\text { OAHI }\end{array}$ \\
2 & 18 & M & 1.6 & 1.4 & 0.8 \\
3 & 13 & M & 3.5 & 10.4 & 1.7 \\
4 & 13 & M & 5.7 & 13.4 & 2.4 \\
5 & 9 & F & 100.5 & 83.1 & 126.2 \\
6 & 16 & M & 3.2 & 6.2 & 5.8 \\
7 & 9 & M & 3.1 & 4.2 & 3.1 \\
8 & 12 & M & 1.8 & 3.1 & 1.7 \\
9 & 10 & M & 12.1 & 21.4 & 19.3 \\
10 & 9 & F & 2.5 & 3.5 & 1 \\
11 & 10 & F & 6.1 & 3.9 & 7.2 \\
12 & 14 & F & 28.1 & 35.8 & 33.6 \\
13 & 9 & $\mathrm{~F}$ & 3.2 & 2.2 & 0.5 \\
14 & 15 & M & 1.9 & 3 & 1.2 \\
15 & 11 & M & 0.8 & 1.1 & 0.6 \\
16 & 14 & M & 24.4 & 35.6 & 28.5 \\
17 & 17 & F & 0 & 0 & 0 \\
18 & 17 & F & 5.8 & 4.8 & 0.5 \\
19 & 16 & F & 0 & 0 & 0.2 \\
20 & 13 & F & 3 & 2.2 & 1.3 \\
21 & 17 & F & 1 & 2 & 0.7 \\
22 & 16 & M & 6.4 & 11.1 & 3.3 \\
23 & 16 & M & 38.9 & 60.3 & 52.6 \\
24 & 10 & M & 1 & 0.4 & 0.3 \\
25 & 13 & M & 1.7 & 1.9 & 1 \\
& 11 & M & 0.9 & 0.9 & 0.6 \\
\hline & & & & &
\end{tabular}

OAHI across the subject pool. The PSG revealed a mean OAHI of $11.8 \pm 27.1$ events/h (range 0-26). Assuming a cutoff OAHI of 5 events/h, we classified 7 of the 25 subjects (28\%) as having moderate to severe OSAS.

Table 2 presents the OAHI values obtained from each modality for each subject. An analysis of correlation (Figure 1) demonstrated that the results from the device autoscore and the PSG were highly correlated (Spearman Rho $=0.886$ $[\mathrm{p}<0.001])$. Table 3 presents the sensitivity, specificity, AUC, AUC standard error, $p$ value, PPV, and NPV for the diagnosis of OSAS obtained from PSG compared with ApneaLink autoscore and manual score at an OAHI cutoff of 1.5, 5, and 10. Using PSG derived diagnoses as the standard, the device autoscore was highly accurate in detecting OSAS, based on high AUC values at each OAHI cutoff tested (Table 3A, Figure 2). The device was most accurate when an OAHI of 10 was used to diagnose OSAS (AUC $1.00[95 \% 0.863,1.00]$ ). The sensitivity of the device in diagnosing OSAS was $100 \%$ at OAHI $>1.5$, $85.7 \%$ at $\mathrm{OAHI}>5$, and $100 \%$ at $\mathrm{OAHI}>10$. A significant number of false positives occurred at $\mathrm{OAHI}>1.5$, as evidenced by a specificity of only $46.2 \%$. However, the specificity of the device increased with application of higher cutoffs (Table 3).

We next asked whether the accuracy of the ApneaLink changes when apnea and hypopnea events are scored manually based on pediatric scoring criteria. We again found a high AUC when manually scored ApneaLink data were compared to PSG 
(Table 3B, Figure 2). Pairwise comparison of ROC curves at each OAHI cutoff did not reveal a significant difference between the ApneaLink auto score and manual score (Table 4).

The ApneaLink also records respiratory effort, allowing obstructive and central events to be distinguished. We used Spearman Rho to correlate the total number of central apneas identified on PSG with the number identified with the ApneaLink autoscore. The absolute number of PSG scored central apneas correlated with the central apneas scored on the Apne$\operatorname{aLink}(\mathrm{r}=0.527, \mathrm{p}=0.007)$.

\section{DISCUSSION}

In this study, we evaluated the utility of using a multichannel portable device to screen for OSAS in obese adolescents. We found that the device has a high negative predictive value for ruling out OSAS at all OAHI cutoffs examined and that the device accurately diagnoses OSAS. Automatic scoring using

Figure 1-Spearman Rho correlation comparing the obstructive apnea hypopnea index of the ApneaLink autoscore with polysomnography

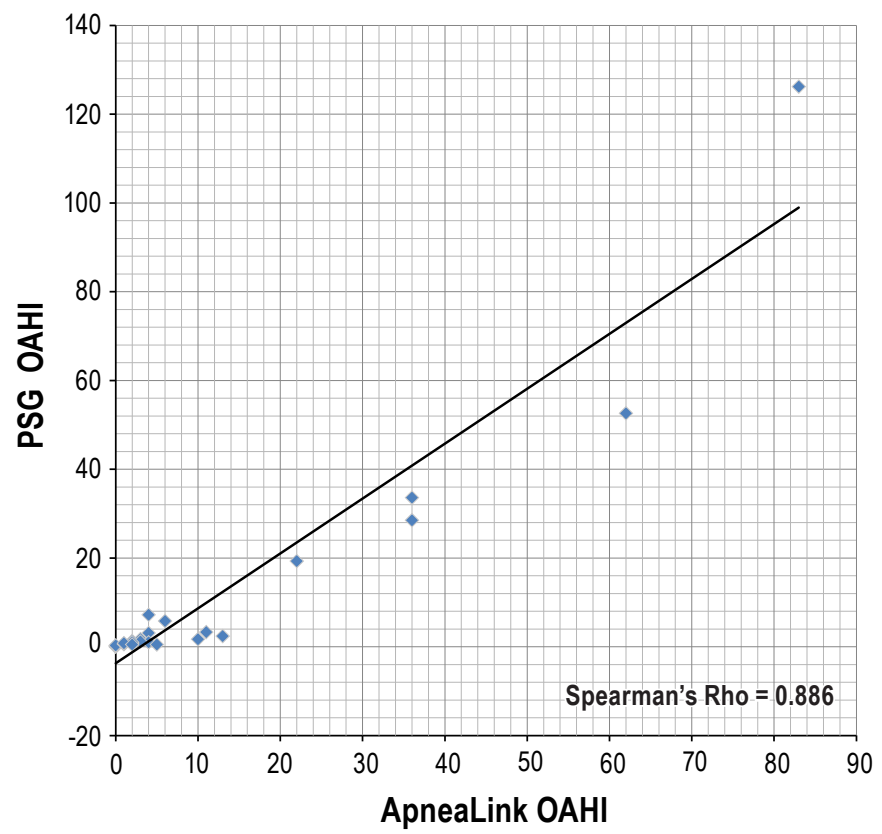

the device software detects OSAS as accurately as does manual scoring. Thus, the portable device detects OSAS to a high degree when tested on obese adolescents referred for symptoms of sleep related breathing disorder.

This result is consistent with those of previous studies that tested portable recording devices in adult subjects. ${ }^{5-12}$ Erman et al. reported an AUC of 0.863 at an $\mathrm{AHI} \geq 5$ and an AUC of 0.862 at AHI $\geq 10$ when comparing the ApneaLink to PSG among 59 subjects with a mean age of 57 years. ${ }^{5}$ In addition, Rofail et al. compared a single-channel device to PSG and found a sensitivity of $94 \%$ and specificity of $62 \%$ at an OAHI cutoff of ten. ${ }^{11}$ The majority of these studies report improved accuracy at OAHI cutoffs of ten and above.

Although consensus has not been reached regarding what pediatric OAHI cutoff for diagnosing OSAS is clinically relevant, the number used by most pediatric sleep laboratories is often less than that used for adults. ${ }^{14,18,20,21}$ Therefore, we tested the device at lower cutoffs and chose to examine the accuracy at OAHI cutoffs of 1.5, 5, and 10. At an OAHI cutoff of 1.5, the sensitivity was $100 \%$. Thus, our data suggest that a normal OAHI identified on an ApneaLink study can likely rule out the diagnosis of OSAS. Autoscore sensitivity decreased to $85.7 \%$ at an OAHI cutoff of 5, but increased to $100 \%$ when an OAHI cutoff of 10 was used. A significant number of false positives $(28 \%)$ were identified when an OAHI cutoff of 1.5 was used. The ApneaLink OAHI was $\leq 5$ in all seven of the false positives identified (Table 2). We therefore suggest that if a sleep lab uses an OAHI cutoff of 1.5, an ApneaLink result between 1.5 and 5 should be referred for PSG. Using this criterion, only 6 of 25 subjects (24\%) would have been ruled out for OSAS with the portable monitor.

However, if an OAHI $<5$ is used as a cutoff for recommending treatment, referrals for PSG could be significantly reduced. We found that 15 of $16(94 \%)$ subjects with an OAHI $<5$ on the ApneaLink autoscore also had a PSG OAHI $<5$. These subjects would not require referral for PSG if an OAHI $<5$ is used to diagnose OSAS. The drawback to this scenario is that patients with PSG OAHI 1.5 to 5 , sometimes identified by pediatric laboratories as having mild OSAS, would go undiagnosed. Outcomes-based research is needed to identify the long-term consequences of leaving such patients untreated. At a minimum, therapy for weight control and counseling that OSAS

Table 3-Sensitivity and specificity of the ApneaLink OAHI compared with PSG OAHI

\begin{tabular}{|c|c|c|c|c|c|c|c|c|}
\hline \multicolumn{9}{|c|}{ A. Autoscore } \\
\hline OAHI & Sensitivity & Specificity & AUC & S. E. & $p$ value & $95 \% \mathrm{Cl}$ & PPV & NPV \\
\hline$>1.5$ & 100 & 46.2 & 0.965 & 0.03 & $<0.001$ & 0.804 to 0.999 & 0.63 & 1.0 \\
\hline$>5.0$ & 85.7 & 83.3 & 0.937 & 0.05 & 0.001 & 0.762 to 0.995 & 0.67 & 0.94 \\
\hline$>10.0$ & 100 & 90.0 & 1.00 & $<0.01$ & 0.001 & 0.863 to 1.00 & 0.71 & 1.0 \\
\hline \multicolumn{9}{|c|}{ B. Manual score } \\
\hline OAHI & Sensitivity & Specificity & AUC & S. E. & $p$ value & $95 \% \mathrm{Cl}$ & PPV & NPV \\
\hline$>1.5$ & 100 & 46.2 & 0.926 & 0.05 & $<0.001$ & 0.748 to 0.992 & 0.63 & 1.0 \\
\hline$>5.0$ & 85.7 & 83.3 & 0.944 & 0.05 & 0.001 & 0.774 to 0.997 & 0.67 & 0.94 \\
\hline$>10.0$ & 80.0 & 100 & 1.00 & $<0.01$ & 0.001 & 0.863 to 1.00 & 1.0 & 0.95 \\
\hline
\end{tabular}

AUC, area under the receiver operator curve; S.E., p value, 95\% CI refer to AUC; PPV, positive predictive value; NPV, negative predictive value. 
Figure 2-Receiver operator characteristics (ROC) curves of OAHI ApneaLink manual and auto score vs. polysomnography at $\mathrm{OAHI}>1.5(\mathrm{~A}), \mathrm{OAHI}>5(\mathrm{~B})$, and $\mathrm{OAHI}>10$ (C)

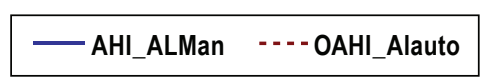

A

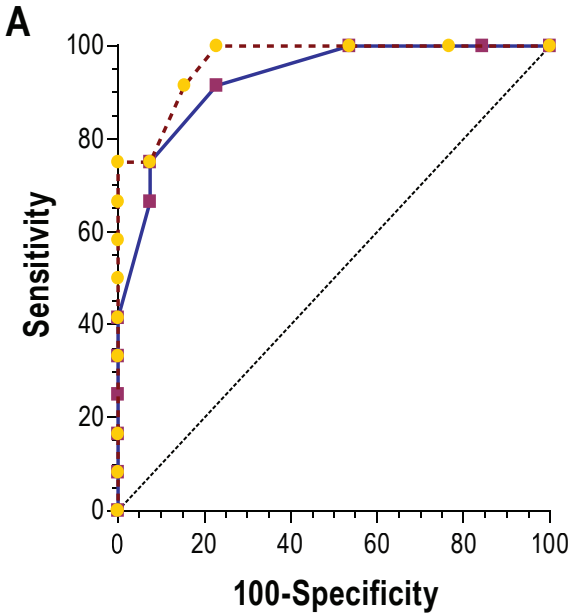

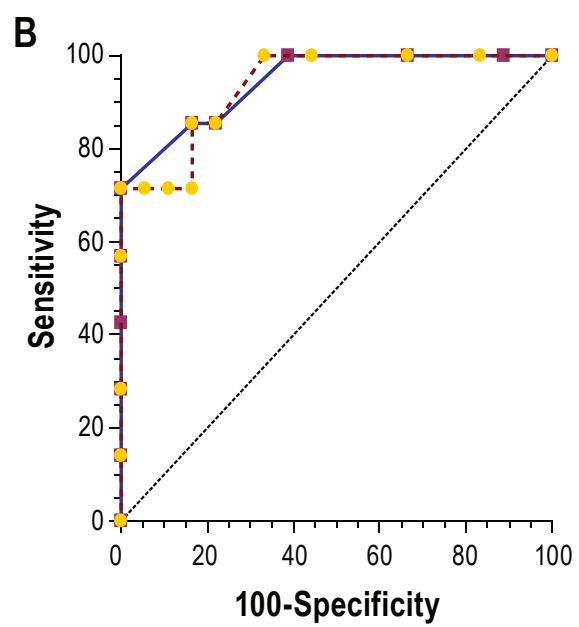

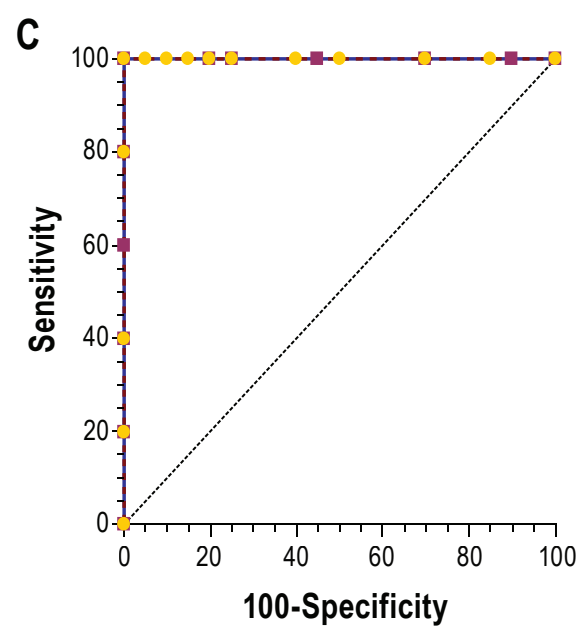

AHI_ALMan, obstructive apnea hypopnea index of the manually scored ApneaLink; AHI_ALauto, obstructive apnea hypopnea index of the automatically scored ApneaLink.

may worsen with continued weight gain for obese adolescents who snore is appropriate.

In addition, all five subjects with an ApneaLink OAHI $>20$ had an OAHI $>20$ identified on PSG. We suggest that subjects identified as having severe OSAS on the portable monitor may be more rapidly referred for therapy when compared with wait times in many laboratories to obtain standard PSG. If positive airway pressure (PAP) is indicated, the use of the portable device to establish the initial diagnosis potentially decreases the need for a baseline PSG and expedites referral for PAP titration. If tonsillectomy and adenoidectomy is performed, then the portable device may be applied to screen for persistent OSAS postoperatively. This indication may also decrease the need for PSG. Before follow-up portable monitoring studies are performed, investigation of test-retest validity of the device in pediatric patients should be established. We believe that if the device can be used successfully in the home setting, it will decrease the need for referral for PSG.

We also found that, compared to interpretation of PSG data, the portable device can overestimate the OAHI (Table 2), consistent with some previous reports. ${ }^{11}$ This overestimation may possibly occur if the device scores obstructive events that would not be scored on PSG, including events not associated with an arousal or desaturation, events scored during periods of wakefulness, or post-arousal artifactual decreases in airflow. ${ }^{11}$ Underestimation of the OAHI may also occur due to the number of apneas being divided by total recorded time rather than total sleep time. ${ }^{22}$ Thus, it would be preferable to have a method of assessing wakefulness with EEG or actigraphy. This will likely increase the validity and reproducibility of portable devices' diagnostic capabilities. In this study, the device's tendency to overestimate the OAHI became more significant at the 1.5 cutoff. However, when an OAHI cutoff $>$ 10 was used, the specificity improved to $90 \%$, suggesting that
Table 4-Pairwise comparison of device autoscore vs. manual score ROC curves

$\begin{array}{cccc}\text { OAHI } & \begin{array}{c}\text { Difference Between } \\ \text { Areas Under Curve }\end{array} & 95 \% \mathrm{Cl} & \text { p value } \\ >1.5 & 0.039 & -0.018 \text { to } 0.094 & 0.18 \\ >5.0 & 0.0079 & -0.067 \text { to } 0.083 & 0.84 \\ >10.0 & 0.000 & 0.000 \text { to } 0.000 & 1.0\end{array}$

the device can identify the overwhelming majority of adolescents with severe OSAS.

The ApneaLink device uses an automatic scoring algorithm based on adult scoring criteria to identify obstructive events. The 2007 American Academy of Sleep Medicine Consensus scoring manual states that children age 13-18 years may be scored by adult or pediatric criteria. ${ }^{19}$ Although some evidence suggests that adult criteria may be applied to asymptomatic subjects 8-18 years, it is likely that the criteria used for scoring obstructive events impacts the interpretation of the test. ${ }^{14,23}$ We therefore rescored the ApneaLink device's output by scoring obstructive events based on respiratory cycle, rather than absolute time. Since the portable device tested does not have EEG, arousal was not used as a criterion for scoring obstructive events. Although the actual number obtained from the manual score differed from the autoscore (Table 2), this did not have a significant impact on the ability to diagnose OSAS. Therefore, the adult scoring criteria used by the device's software may be acceptable for screening purposes when testing symptomatic adolescent patients.

The automatic scoring output of the device includes central apnea in its calculation of the AHI. For this study, the AHI was recalculated to reflect only obstructive events. The pathophysiology, diagnostic workup, and therapy of pediatric central sleep apnea syndromes differ significantly from those of OSAS. 
Differentiation of patients with central sleep apnea is thus an important function of a screening device. We found that the absolute number of central apneas identified by the ApneaLink autoscore correlated well with that found on PSG. However, because central apnea was not the primary outcome tested for this study, and we did not enroll patients with central apnea syndromes, we cannot comment on the utility of the device in detecting pediatric central sleep apnea.

There is currently limited data regarding the use of screening devices in the pediatric population. ${ }^{15-18,24}$ We chose our study population based on the high incidence of pediatric obesity, with the aim of addressing the need for screening symptomatic individuals for OSAS. A number of unique features and limitations deserve comment. The study population was recruited from subjects referred to a tertiary pediatric center, thus likely accounting for the relatively high incidence and severity of OSAS observed. It should be noted that the investigator who manually scored the ApneaLink was not blinded to the result of the PSG and may have been biased by the PSG result. At the time of this study, our sleep laboratory used a thermistor to monitor airflow. Thermistors are known to have low sensitivity for detecting hypopnea, and thus the degree of OSAS may have been underestimated on the PSG. ${ }^{25}$ Nevertheless, Nakano et al. did not find that using a thermistor vs. nasal pressure transducer for the detection of airflow on PSG significantly affected their ability to estimate the diagnostic utility of a single-channel airflow device. ${ }^{26}$

Most of our enrolled subjects (25 of 29; 86\%) had at least two hours of usable data recorded on the device, a result similar to previous reports. ${ }^{5,11}$ Less than two hours of recorded time is likely inadequate for diagnosing OSAS, especially if REM sleep is not captured. On average, over two hours of recording time was automatically removed by the device due to inadequate airflow (Table 1). We observed a large degree of variability in the amount of time removed, as evidenced by a large standard deviation and wide range. Much of the removed data may have been due to poor signal related to displacement of the nasal canula or possibly nasal obstruction. The flow evaluation time and percent flow data removed are important variables for practitioners to consider when assessing the validity of any portable study. However, although the device had less usable data available for scoring apnea than the PSG, this did not appear to affect its ability to diagnose OSAS. It is also important to highlight that this study simultaneously compared the ApneaLink device to PSG in the sleep laboratory. Thus, it is not known if the results can be translated to the home setting. Previous studies have found agreement between the sleep laboratory and home environments. ${ }^{5,10}$ Although pediatric patients may have more difficulty tolerating the device than adults, other pediatric studies have suggested that home testing is feasible in the research setting. ${ }^{17,24,27}$

In summary, the portable device tested in this study accurately identifies OSAS when tested in a sleep laboratory on obese adolescents referred for symptoms of sleep related breathing disorder. Although the device is sensitive even at low OAHI cutoffs, the specificity increases when higher OAHI cutoffs are used. We speculate that the device can be used to screen obese adolescents for the presence of OSAS. The decision to refer for standard PSG may depend on the OAHI cutoff used by the clinician. Investigation of the accuracy of the device when used in the home and when applied to other pediatric populations will help to define further the appropriate use of multichannel screening devices in pediatric patients.

\section{REFERENCES}

1. Han JC, Lawlor DA, Kimm SY. Childhood obesity. Lancet 2010;375:1737-48.

2. Gozal D, Crabtree VM, Sans Capdevila O, Witcher LA, Kheirandish-Gozal L. C-reactive protein, obstructive sleep apnea, and cognitive dysfunction in schoolaged children. Am J Respir Crit Care Med 2007;176:188-93.

3. Amin R, Somers VK, McConnell K, et al. Activity-adjusted 24-hour ambulatory blood pressure and cardiac remodeling in children with sleep disordered breathing. Hypertension 2008:51:84-91.

4. Redline S, Storfer-Isser A, Rosen CL, et al. Association between metabolic syndrome and sleep-disordered breathing in adolescents. Am J Respir Crit Care Med 2007:176:401-08.

5. Erman MK, Stewart D, Einhorn D, Gordon N, Casal E. Validation of the ApneaLink for the screening of sleep apnea: a novel and simple single-channel recording device. J Clin Sleep Med 2007;3:387-92.

6. Grover SS, Pittman SD. Automated detection of sleep disordered breathing using a nasal pressure monitoring device. Sleep Breath 2008;12:339-45.

7. Chen $\mathrm{H}$, Lowe AA, Bai Y, Hamilton P, Fleetham JA, Almeida FR. Evaluation of a portable recording device (ApneaLink) for case selection of obstructive sleep apnea. Sleep Breath 2009;13:213-19.

8. Ng SS, Chan TO, To KW, et al. Validation of a portable recording device (ApneaLink) for identifying patients with suspected obstructive sleep apnoea syndrome. Intern Med J 2009;39:757-62.

9. Ragette R, Wang $Y$, Weinreich $\mathrm{G}$, Teschler $\mathrm{H}$. Diagnostic performance of single airflow channel recording (ApneaLink) in home diagnosis of sleep apnea. Sleep Breath 2010;14:109-14.

10. Rofail LM, Wong KK, Unger G, Marks GB, Grunstein RR. The utility of singlechannel nasal airflow pressure transducer in the diagnosis of OSA at home. Sleep 2010;33:1097-105.

11. Rofail LM, Wong KK, Unger G, Marks GB, Grunstein RR. The role of singlechannel nasal airflow pressure transducer in the diagnosis of OSA in the sleep laboratory. J Clin Sleep Med 2010;6:349-56.

12. Oktay B, Rice TB, Atwood CW Jr, et al. Evaluation of a single-channel portable monitor for the diagnosis of obstructive sleep apnea. J Clin Sleep Med 2011;7:384-90.

13. Rosen CL, D'Andrea L, Haddad GG. Adult criteria for obstructive sleep apnea do not identify children with serious obstruction. Am Rev Respir Dis 1992:146:1231-34

14. Accardo JA, Shults J, Leonard MB, Traylor J, Marcus CL. Differences in overnight polysomnography scores using the adult and pediatric criteria for respiratory events in adolescents. Sleep 2010;33:1333-39.

15. Nixon GM, Kermack AS, Davis GM, Manoukian JJ, Brown KA, Brouillette RT. Planning adenotonsillectomy in children with obstructive sleep apnea: the role of overnight oximetry. Pediatrics 2004;113:e19-25.

16. Kirk VG, Bohn SG, Flemons WW, Remmers JE. Comparison of home oximetry monitoring with laboratory polysomnography in children. Chest 2003;124:1702-08.

17. Moss D, Urschitz MS, von Bodman A, et al. Reference values for nocturnal home polysomnography in primary schoolchildren. Pediatr Res 2005;58:958-65.

18. Gozal D, Kheirandish-Gozal L. New approaches to the diagnosis of sleep-disordered breathing in children. Sleep Med 2010;11:708-13.

19. Iber C, Ancoli-Israel S, Chesson A, and Quan SF for the American Academy of Sleep Medicine. The AASM manual for the scoring of sleep and associated events: rules, terminology and technical specification, $1^{\text {st }}$ ed. Westchester, IIlinois: American Academy of Sleep Medicine, 2007.

20. Wong TK. The search on an ideal disease marker for childhood obstructive sleep apnea syndrome. Sleep 2011;34:133-34.

21. Witmans MB, Keens TG, Davidson Ward SL, Marcus CL. Obstructive hypopneas in children and adolescents: normal values. Am J Respir Crit Care Med 2003;168:1540

22. Masa JF, Corral J, Pereira R, et al. Therapeutic decision-making for sleep apnea and hypopnea syndrome using home respiratory polygraphy: a large multicentric study. Am J Respir Crit Care Med 2011;184:964-71.

23. Tapia IE, Karamessinis $L$, Bandla $P$, et al. Polysomnographic values in children undergoing puberty: pediatric vs. adult respiratory rules in adolescents. Sleep 2008:31:1737-44 
24. Goodwin JL, Enright PL, Kaemingk KL, et al. Feasibility of using unattended polysomnography in children for research--report of the Tucson Children's Assessment of Sleep Apnea study (TuCASA). Sleep 2001;24:937-44.

25. Farré R, Montserrat JM, Rotger M, Ballester E, Navajas D. Accuracy of thermistors and thermocouples as flow-measuring devices for detecting hypopnoeas. Eur Respir J 1998:11:1779-82.

26. Nakano H, Tanigawa T, Furukawa T, Nishima S. Automatic detection of sleep-disordered breathing from a single-channel airflow record. Eur Respir J 2007;29:728-36.

27. Verhulst SL, Franckx H, Van Gaal L, De Backer W, Desager K. The effect of weight loss on sleep-disordered breathing in obese teenagers. Obesity (Silver Spring) 2009;17:1178-83.

\section{ACKNOWLEDGMENTS}

The authors thank the Rady Children's Hospital San Diego Center for Pediatric Clinical Research, Serenity Sleep \& Neurodiagnostics, Inc for provision of staff and expertise, and Dr. Jeffrey Schwimmer for referral of subjects.

\section{SUBMISSION \& CORRESPONDENCE INFORMATION}

Submitted for publication September, 2011

Submitted in final revised form December, 2011

Accepted for publication December, 2011

Address correspondence to: Daniel J. Lesser, M.D., Division of Respiratory Medicine, Department of Pediatrics, University of California, San Diego, Rady Children's Hospital-San Diego, 3020 Children's Way MC 5070, San Diego, California 92123; Tel: (858) 966-5846; Fax: (858) 569-9052; E-mail: dlesser@ucsd.edu

\section{DISCLOSURE STATEMENT}

This work was supported by a research grant from the ResMed Foundation. The authors independently analyzed the data and prepared the manuscript. Dr. Lesser has received research funding from ResMed. Drs. Haddad, Bush, and Pian have no other conflicts of interest. The ApneaLink device studied is indicated for adults and is thus under investigational use for pediatric patients. 